\title{
Age period cohort analysis in cancers associated with overweight and obesity in Ireland
}

\section{Abstract}

The Central Statistics Office (CSO) in Ireland report that $62 \%$ of adults were overweight or obese in $2017^{(1)}$. In 2019 , the latest data from the national longitudinal study "Growing Up in Ireland" showed that $15 \%$ of five-year olds were overweight and 5\% were obese $^{(2)}$. According to WHO projections, Ireland may have the highest prevalence of overweight and obesity in Europe by $2030^{(3)}$. The International Agency for Research on Cancer (IARC) state there is sufficient evidence for a cancer preventative effect of the absence of excess body fatness for 13 different cancer sites ${ }^{(4)}$. Recent USA based research showed that the risk of developing an obesity-related cancer seems to be increasing in a stepwise manner in successively younger birth cohorts ${ }^{(5)}$, therefore we aimed to investigate trends in the age of obesity related cancer diagnosis.

National Cancer Registry Ireland (NCRI) data was obtained for 25 to 84 year olds between 1997 and 2016 for several cancer types and 5-year intervals were constructed to categorise age (25-29, 30-34 etc.) and year of diagnosis (1997-2001, 2002-2006 etc.). An age-period-cohort model was fitted to each cancer type, resulting in estimated temporal trends in incidence (expressed as annual percent change (APC) per year) and significance of the trend across age groups was tested using a Wald test ${ }^{(5)}$.

Analysis of NCRI data reflect significantly increasing incidence of colorectal cancer with decreasing age in Ireland $(\mathrm{n}=40703, \mathrm{p}=$ 0.00). Despite the limitation of low numbers for several cancer sites in younger age groups, similar trends were observed for a number of cancer types including thyroid and cervical cancer $(\mathrm{p}<0.05)$. Although other factors (such as early detection) may contribute to these observations, addressing the large increase in incidence of obesity and colorectal cancer among young adults is essential. Research to identify exposure to modifiable risk factors, including nutrition and physical activity, throughout life in Ireland is necessary to examine potential determinants of such trends.

\section{Conflict of Interest}

There is no conflict of interest

\section{References}

1. Central Statistics Office. (2018) Ireland's Facts \& Figures.

2. Economic \& Social Research Institute. (2019) Growing Up in Ireland: The Lives of 5 yo's.

3. WHO (2013) Europe. Nutrition, Physical Activity \& Obesity Ireland.

4. Lauby-Secretan et al. (2016) Body Fatness \& Cancer- Viewpoint of the IARC Working Group. $N$ Engl J Med.

5. Sung et al. (2019) Emerging cancer trends among young adults in the USA: analysis of a population-based cancer registry. Lancet Public Health. 